\title{
A Scoreboard to evaluate Clusters' COMPETITIVENESS IN THE KNOWLEDGE-BASED ECONOMY. AN EMPIRICAL STUDY ON EMILIA ROMAGNA REgION
}

\section{ANDREa Ganzaroli \\ GianluCA Fiscato \\ LuCiano Pilotti}

Working Paper n. 2006-30

OTTOBRE 2006

Scienze Economiche, Aziendali e Statistiche

Via Conservatorio 7 -- 20122 Milano

tel. ++390250321501 (21522) fax ++390250321450(21505)

http://www.economia.unimi.it E Mail: dipeco@unimi.it 


\title{
A scoreboard to evaluate clusters' competitiveness in the knowledge-based economy. An empirical study on Emilia Romagna region.
}

\author{
Andrea Ganzaroli \\ Email: andrea.ganzaroli@unimi.it \\ Gianluca Fiscato \\ Email: gianluca.fiscato@ncl.ac.uk \\ Luciano Pilotti \\ Email: luciano.pilotti@unimi.it
}

Department of Economics, management and Statistics (DEAS)

Faculty of Political Science

University of Milan

Via Conservatorio, 7

20122 - Milan (Italy)

Tel. +39-02-50321530 


\title{
A scoreboard to evaluate clusters' competitiveness in the knowledge-based economy. An empirical study on Emilia Romagna region*.
}

\author{
Andrea Ganzaroli ${ }^{\dagger}$, Gianluca Fiscato and Luciano Pilotti
}

This revision: September 2006

\begin{abstract}
Our main objective with this paper is to propose a scoreboard useful to assess clusters' competitiveness in a knowledge-based economy. Our scoreboard is grounded on the concept of ecology of value. This concept highlights that the competitiveness of a network of small and medium enterprises depends on its capacity to leverage on firms' strategic autonomy and self-determinacy by providing a context of interaction that is socially rich and adapted to sustain the development of intense ongoing relationships. In our scoreboard the concept of competitiveness is structured into two levels. The top level is made of a matrix that synthesizes cluster's competitive position in terms of quality of the social capital available and of the learning process activated. The second level is made of three matrixes useful to deepen the reasons that contribute to define a cluster's competitive position. We test our scoreboard in seven clusters of Emilia Romagna. Our scoreboard proves itself a useful benchmarking tool and a potential good support for policymaking.
\end{abstract}

JEL Classification: L22, L25, L53, O18

Keywords: cluster, competitive advantage, knowledge creation, scoreboard

\footnotetext{
We are grateful to Ecipar to provide the database and the research facilities.

† Andrea Ganzaroli

andrea.ganzaroli@unimi.it

Department of Economics, Business and Statistics

University of Milan

Via Conservatorio, 7 ( $2^{\text {nd }}$ floor - Room 20)

20122 - MILANO (Italy)

Phone office:

Direct: +39-02-503-21530

Department Secretary: + 39-02-503-21522

Department Fax: + 39-02-503-21505
} 


\section{Introduction}

Our main objective with this paper is to present a scoreboard useful to evaluate clusters' competitiveness in a knowledge-based economy ${ }^{1}$. Our understanding of competitiveness is grounded on the notion of ecology of value (Arthur, 1996; Ganzaroli \& Pilotti, 2006; Iansiti \& Levien, 2004; Pilotti \& Rinaldin, 2004). This concept, as we shall see, highlights that the competitiveness of a network of small and medium enterprises depends on its capacity to leverage on firms' strategic autonomy and selfdeterminacy by providing a context of interaction that is socially rich and adapted to sustain the development of intense ongoing relationships. This implies a shift of perspective from the traditional way of looking at clusters. This field of literature, in fact, points out long-term, trusted and local relationships as strategic for local systems' survival. Our concept of ecology, differently, shows that these kind of relationships are compatible with clusters' sustainable development if and only if accompanied by a high level strategic autonomy. This is a precondition, on the one hand, to enlarge learning capacity and, on the other, to prevent trust from diverting into collusion due to the progressive interlocking between reciprocal weaknesses and constrains.

Our scoreboard is structured into two levels. At the top one there is a matrix that summarizes clusters' competitive position. It is based on two dimensions. The first addresses the quality of the social capital available in the cluster defined as combination between intensity and extensity of the relationships. The second refers to the quality of the learning process between learning by doing and learning by interacting. The more the local system is positioned on the top part of the matrix - high intensive and extensive relationship and interactive process of knowledge creation the more its development is sustainable.

The second level is defined by three matrixes, which address most of the factors that contribute to explain clusters' competitive capacity. The first addresses the relationship between firms' relational intensity and firms' strategic autonomy. This matrix enables us to evaluate to which extent firms in the local network behave ecologically. This means that are capable to mobilize external resources to their own advantages without exhausting them. This implies that their behavior is not oriented toward the maximization of actual profit but to the regeneration of competitive resources in the local system. The second measures the relationship between type of activities developed internally and quality of the competencies available. On the base of these two dimensions we are capable to identify the quality of the learning process activated by firms in the cluster. Learning is based mainly on experience in firms that control only a small part of the entire process and do not hold strategic capabilities. Leaning is mainly interactive in firms that coordinate a large part of the supply chain and hold the an extensive base of capabilities. The last matrix, in the end, link the sustainability of the process of firms' development to the cluster's perceived role. This is useful to understand to which extent the cluster is still perceived as a source of competitive advantage for the firm.

We argue that this scoreboard is extremely versatile because enable us to express evaluation both at aggregate level - at the level of local system - and at the one of single firm. We suggests that this system is particularly useful for local policy maker to monitor the effect of their investments and activities in the system. This system, as we shall see, is meant to develop a benchmarking analysis between local systems. Therefore, it is a trans-local learning infrastructures that should enable the development cooperative and interactive learning between policy makers. Therefore, we also address the need to develop a transnational infrastructures to enable cross-

\footnotetext{
${ }^{1}$ In this paper we refer to the concept of cluster as synonymous of industrial district according to authors as Maskell (2001), Asheim and Isaken (2002) and Cooke and Huggins (2002). For a review about the concept of cluster see Martin and Sunley (2003) and Belussi (2005).
} 
cultural and cross-local learning. Finally, in order to show how the system's work, we also provide a case of benchmarking between seven clusters in Emilia Romagna.

The paper is structured into three parts. The first part is devoted to provide the reader with the theoretical background useful on which our scoreboard is grounded on. In the second part we address the methodological aspects. We define the sampling method and the characteristic of the sample. We describe the questioner and data collection. We discuss the limits of our methodology and how we tried to overcome them. Finally, the third part is devoted to the case study, where we develop a benchmark among seven clusters in Emilia Romagna. In the conclusive section we summarize our major findings and limitations and we discuss future research directions.

\section{Clusters' competitiveness: Toward an ecological perspective}

The concept of clusters' competitiveness has been largely debated since Porter's "The Competitive Advantage of Nation" (1990) (Boschma, 2004). Porter, in this contribution, recognizes "regions" as potential source of competitive advantage. According to his perspective, in fact, localities may embed peculiar competitive resources, which are historical produced through the specific way market and regulative forces interact in place. It should be noted that, according to Porter, the competitive advantage of nation is still traced back to the peculiar way market forces are combined and sediment in one place. Therefore, Porter does not position himself outside the neoclassical tradition, which explains the development of international trade as consequence of comparative advantages/disadvantages between nations. However, Porter's contribution has been to suggest the possibility to extend the applicability of strategic tools to the analysis of regional competitiveness providing a sort of common language to enhance the cooperation between public and private sectors in the management of public or quasi-public resources.

Even if the concept of regional competitiveness has became commonly accepted, there are scholars, such as Krugman (1994), that see a potential danger on extending its application to regional governance. In his view, in fact, regions do not compete as firms' do. They do not enter and exit markets. Their scope is not overwhelm their competitors and so forth. These differences, according to Krugman (1994), may have an impact of the way governments perceive their role with the consequence of leading to a wasteful use of public resources and bad policymaking ${ }^{2}$. Furthermore, it may also lead to a reemergence of a protectionist attitudes justified by the need to protect national competitive advantages. Even if Krugman's arguments are partially shareable, we believe that the development of information, communication and transportation technologies, on the one hand, and the dematerialization of production and consumption, on the other, has brought regions to compete on their capacity to attract and withhold highly mobile competitive resources, such as knowledge and creativity 3. Therefore, these perspective may prove itself useful to enhance government strategic capacity and cooperation between private perspective.

There are a number of theory that attempts to explain why region keeps their attractiveness despite of the wide diffusion of information and communication technologies ${ }^{4}$. There are authors, such as Krugman (1991), that argue that the competitiveness of places is simply consequence of the particular way market forces have localized and sediment in one place in the broader context of international trade. Therefore, the competitive advantage of nations is simply a comparative advantages

\footnotetext{
${ }^{2}$ Krugman argues that politicians have welcomed the adoption of these tools because it has enable them to shift public attention form their specific responsibility and the need to reform the social state.

${ }^{3}$ See Florida (2002; , 2005) for an emergent perspective.

${ }^{4}$ See for a recent review Newlands (2003).
} 
that has localized in one place because of historical chances. Others have argued that the competitive advantage of places can be traced back to existence of un-traded interdependencies. Therefore, being spatially clustered enable firms to minimize on transaction costs associated to the governance of quasi-public resources. A third perspective - flexible specialization - focuses on the issue of trust. Therefore, spatial proximity, mainly as consequence of cultural homogeneity, enables firms to save on the costs of trust production and knowledge transfer between firms. A fourth perspective - the milieu innovator/cognitive approach - see regional competitiveness as consequence of improved collective learning capacity. Local capacity to attract and withhold high value economic resources and activities depends on the quality of the framework for learning that localities itself provide. Finally, a fifth perspective evolutionary - sees regional development as the spatial clustering of a collective and highly irreversible process of decision making. This perspective share the same focus as the previous (local learning and innovation). However, it is grounded on concepts such as increasing returns, network externalities and lock-in. The process of regional development is considered to be path dependent and regions, as collective and complex units of decision making, may position themselves along inferior technological and learning trajectories. Therefore, the main problem that evolutionist attempts to solve is to define the governance structure that enable region to remain open to emerging technological and learning trajectories.

Our analysis of regional competitiveness is grounded on the notion of evolutionary ecologies of values. Therefore, we position ourselves in the evolutionary field of literature. The arguments that justify our decision is that we are strongly convinced that nowadays regional competitiveness and development strictly depend on the capacity of local institutions and firms to govern openness. Nowadays the local and national systems are incapable to self-produce the knowledge necessary to compete in a global market. Therefore, these systems are required, on the one hand, opening up themselves to global networks of knowledge creation, transfer and diffusion and, on the other, specializing themselves on specific activities along these cognitive supply chains. From this perspective, local competitive resources and competencies - such as cultural homogeneity, trust and a prevalence of manual abilities - that have made the fortune of these systems in the past become core rigidity, which prevent local systems from enlarging their base of innovation and knowledge.

However, in our perspective, local system are not only evolutionary, but also ecological. The use of the term ecology is not new to the managerial literature5. It identifies a specific filed of study, which is grounded on the hypothesis that evolution in a population of firms - such as in an industry and/or market - does not take place through organizational change, but market selection. Successful firms, according to this perspective, are affected by organizational inertia. Success, in fact, inhibits firms capacity to perceive and interpret change. Furthermore, even if this is perceived and understood, managers do not hold enough power to contrast the organizational inertia and enforce the relative organizational solutions. Therefore, the lost of competitiveness by consolidated firms makes room to new ones, which are technologically and organizationally more advanced, that progressively overwhelm the established ones. Therefore, it is market selection and not organizational change the main driver of innovation and renovation in an industry/market ${ }^{6}$.

The ecological perspective as such is compatible with the study of regional competitiveness. The population corresponds with the local firms and institutions. From this perspective, ecologist would argue that regional competitiveness depends on the number of firms entering and exiting the local market. It is, in fact, by the means of this process that the local systems renovates it competitive base and keeps it

\footnotetext{
${ }^{5}$ See Baum et. al. (2006) for a recent review on the subject.

${ }^{6}$ It should be noted that from this perspective local institutions should not stimulate existing firms to adopt new technologies, but the birth of new firms.
} 
technologically updated. However, this perspective has a major limit. It is anchored to a darwinistic view of evolution. In this perspective evolution is the outcome of the natural selection of those genetic mutations that have proven themselves more efficient in a given environment. Therefore, evolution takes place through an error in the recombination and transmission of the genetic pool from one generation to the following one. The major limit of this way of looking at evolution is that it does not take into account the network of reactions and feedbacks that these mutations enact. On the contrary, if this network is taken into consideration, we discover that the nature does not select beings that have proven themselves most competitive and effective in a given environment, but the ones that have proven themselves more faithful and loyal to the environment they are embedded in. This means that they have been capable to construct a network of complementarities - collaborations and antagonisms - that is compatible with the reproduction and evolution of the ecology (Morin, 1980).

The major advantage of this second perspective is that it recognizes creativity as a process endogenous to an ecology. Evolution, in fact, is not anymore only the result of a casual mutation occurred in the transmission of genetic pool. Therefore, it is not anymore sufficient to increase the rate of reproduction in order to sustain innovation. The quality of the ecosystem and indeed of the cluster as common ground for learning and knowledge creation is strategic to enable firms' capacity to absorb, create and transfer knowledge.

The creativity of an ecology, according to Morin (1980), is tied to four major aspects. The first is resource variety. Ecologists use the concept of biodiversity in order to convey the relevance that a wide variety of resources has for its capacity to survive and evolve. The number of recombination that is potentially producible increases exponentially with the variety of available resources in the ecosystem. The second aspect is connectivity. The probability of recombination increases with the network connectivity and indeed with its capacity to stimulate the spontaneous encounter between diverse resources. The relevance of these two aspects is widely recognized in the literature ${ }^{7}$. Even more it is suggested the existence of a trade off between connectivity and cognitive distance/resource variety. An excess of cognitive distance between participants to a network may inhibit knowledge transfer because of the lack of a common communication-base. This is also recognized by ecologist, who argue that the interaction between any variety of resources does not make an ecology. Ecology are build up on a core network of relationships. Therefore, it is the quality of this core that influence, but do not determine the capacity of the ecology to sustain its development. Form this perspective, openness play a key role. Ecologies that are not capable to exchange and share energy (knowledge) with others are destined to implode as consequence asphyxia. Prigogine argues that creativity is a property that emerges in network dominated by positive feedbacks, which progressively brings the system far from the initial equilibrium, and that are capable to activate connections between previously distant nodes in the network. This second characteristic is required in order to prevent the system from degrading into chaos.

The last aspect that contradistinguishes a creative ecology is freedom. Life springs into ecologies where each component enjoys large freedom to explore its combinatorial potential through the network. Freedom has been recently recognized in economic literature as fundamental for the development of form of reciprocity that are far more complex than the contractual one (Bruni, 2006). Relational goods, such as friendship, may emerge only in context where both parties may choose to enter the relationship with other. The literature, form this perspective, distinguishes between negative freedom and positive freedom (Sen, 1995). The first refers to the freedom from something and/or someone. The second, instead, refers to the freedom to do something. In this second form of freedom, which is the most relevant and complete, the exercise of freedom does not require only the possibility to do something, but also

\footnotetext{
${ }^{7}$ See (Boschma, 2004).
} 
the ability to do it. Therefore, the exercise of positive freedom is grounded on the availability of knowledge and competence. This, as we shall see, is contemplated into our score board to measure cluster competitiveness in the concept of autonomy. Clusters that are capable to better sustain their development are those that integrate high level of social connectivity and individual autonomy, where autonomy is define by the availability of knowledge and competencies necessary to interact in a global network of knowledge creation.

\section{Research Methodology}

This paper reports on the findings of a study of seven clusters located in Emilia Romagna region and differentiated by level of technology and sector. The study investigated the possibilities to create a policy tool, based on benchmarking, useful to assessing the competitiveness of clusters. This instrument takes into account the specific problems faced by a cluster and the specific sources of competitiveness. It is not a "one-size-fits-all" policy tool that puts every cluster in the same trajectory of evolution (Isaksen, 2001). It permits to understand the competitiveness framework and to avoid the use of standard local industrial policies. In conclusion it is a tool, based on benchmarking, useful to define a "tailor-make" policy portfolio (Isaksen, 2001).

\section{The questionnaire}

In order to collect the data we develop a questionnaire structured into two parts. The decision to divide the questionnaire responded to simplify the need to minimize the costs of data collection.

The first part, which contained self-explicatory questions, has been sent by e-mail. Firms have been previously contacted by phone or mail in order to solicit participations and to provide them with a background of the research project and a description of the questionnaire. The first part of the questioner was structured in two sub-parts:

1. Firm's general data (contact details, address, name and address of Managing Director), size of company (number of employees) and economic activity (ATECO ${ }^{8}$ sector);

2. Type of processes/activities carried out by the firm and degree of control retained over it (14 binary items).

The second part, whose compilation may have required some additional assistance, was collected through a series of personal interview to firms' entrepreneurs or managers. This second part was mainly structure into three sub-parts:

1. Relevant information about operations managerial practice and performance (30 items);

2. Assessment of firm's distinctive competence in eight (production management, supplier management, customer management, channel management, technology management, human resource management, financial management and strategic management) different areas (51 items);

3. Relevance and type of links with regional system in comparison to links with global system (13 items).

The first sub-part is based on Frames benchmarking tool ${ }^{9}$. Frames is designed on the hypothesis that firms' sustainable development is constructed on the balance

\footnotetext{
${ }^{8}$ ATECO 2002 is the Italian economic activity classification based on NACE Rev 1.1.

${ }^{9}$ The data collect by Frames' questionnaire enable firms of sample to participate at Regional Excellence Award 2005 and at Regional Report on Competitiveness 2005. However, only a sub-set (8 items) of Frames' items is used in the scoreboard.
} 
between performance and manufacturing, design and business practices. This tool developed by Ecipar gathers information on key firms' strategic dimensions, such as organisation and culture, production, quality, competitive positioning, financialeconomic performances. Responses ranges from 1 to 5, where 1 represents the basic level of practice/performance and 5 the best ${ }^{10}$.

The second and the third sub-parts were linked to a five-point Likert-type scale from "absolutely non important" to "remarkably important". The first draft of the questioner was self-developed on literature and subsequently tested with academics and practitioners.

\section{The scoreboard: objectives and framework}

The main objective of the scoreboard is threefold. The first is to evaluate the competitiveness of cluster characterized by the prevalence of small and medium enterprises and facing the challenges of the knowledge and global-based economy. The second is to deepen our understanding of competitive factors in the knowledgebased economy. The third is to build a benchmarking tool useful for policy makers to monitor the effect of their investments and activities in the system. Therefore, the scoreboard could be a trans-local learning framework useful to enable the development of cooperative and interactive learning between policy makers.

The scoreboard is based on the concept of cluster's competitiveness in an ecology view. According to this view the competitiveness of a cluster depends on its capacity to leverage on firms' strategic autonomy and self-determinacy by providing a context of interaction that is socially rich and adapted to sustain the development of intense ongoing relationships. In the assessment of the cluster's competitiveness and in the development of industrial/local policies is important consider the strategic autonomy. It enables to enlarge learning capacity and to prevent trust from diverting into collusion and lock-in phenomena.

The scoreboard is structured into two levels and four matrixes.

At the first level there is a matrix that summarizes cluster's competitive position of cluster. It is named the Competitiveness Matrix. It is based on two dimensions:

- Quality of Network: it refers to the quality of relationships available in the cluster. It was defined as combination between intensity and extensity of the relationships. This dimension evaluates firms' capacity of cluster to sustain business development by both local networking and strategic autonomy.

- Quality of Learning and Knowledge: it The second refers to the quality of knowledge. It was defined as combination between the capability to carry out process and to acquire and manage new knowledge. This dimension evaluates cluster's capacity to activate high-knowledge processes of interactive learning interaction.

The more the cluster is positioned on the right-top part of the matrix -high quality of learning, knowledge and networking - the more the sources of competitive advantage are sustainable in a knowledge-based economy.

The second level is defined by three matrixes, which address most of the factors that contribute to explain the clusters' competitive capacity. The matrixes at this level measure:

\footnotetext{
${ }^{10}$ The Frames tool was used in some European Project and Frames' database contains more than 3800 firms.
} 
1. The learning system activated in the cluster (The Learning/Knowledge Matrix);

2. The degree of matching between strategic autonomy and networking (The Ecological Network Matrix);

3. The perceived relevance of the local context in sustaining cluster's competitiveness (The Local System Matrix).

The first matrix evaluates the relationship between types of activities carried out internally and quality of the competence available. It is named the Learning/Knowledge Matrix. The matrix is defined by two indexes:

- Capabilities: it is calculated as weighted average of the scores on these aspects: production management, supplier management, customer management, channel management, technology management, human resource management, financial management and strategic management. In other words, it evaluates the distinctive business competencies that belong to firms' cluster.

- Processes/activities internalized: it is calculated as weighted average of processes/activities carried out in the firms. It uses a different weight for the activities and for the degree of control on it. It gives a score to firm's position on value chain.

On the base of these two dimensions it is possible to identify what is the main form of learning in the cluster. Low levels of both dimensions identify a prevalence of learning by doing. Low number of processes and high levels of competences identify the prevalence of learning by imitation. In this case the majority of cluster's firms are specialized in a little number of activities that carried out without control all the process. High levels of both dimensions identify the prevalence of learning by interacting characterized by high-knowledge intensity. The last case - low competences and high number of processes - identify alike the prevalence of learning by imitation but it is characterized by low-knowledge intensity.

The second matrix addresses the matching between firms' relational intensity and firms' strategic autonomy. It is named the Ecological Network Matrix. This matrix enables us to understand to which extent firms in the cluster behave ecologically. In other words, it evaluates if they are capable to mobilize external resources to their own advantages without exhausting them. This implies that their behavior is not oriented toward the maximization of actual profit but to the regeneration of competitive resources in the local system. The matrix is defined by two indexes:

- Strategic Autonomy: it is calculated as difference between the weighted average of the number of activities/processes that the enterprise is able to control and the weighted average of the number of activities/processes that the enterprise is not able to control. It uses a different weight for the activities and for the degree of control on it. It gives a score to firm's strategic autonomy;

- Relational Intensity: it is calculated as mean of the importance attributed by the enterprise to networking practices and alliances with clients and suppliers. In other words, as the geographical proximity only creates a potential for interaction, it evaluates the strategic importance give to networking by firms.

Moving from left to right improves the strategic autonomy and, thereby, the levels of knowledge and competences. Therefore, the firms, moving along the $\mathrm{x}$-axis, improve the degree of specialization and/or specialize in high-value activities. Moving from bottom to up improves the relational intensity and, consequently, changes the type from short-term to long term - and the mode - from transactional to relational mode- of the relationships. Therefore, the density of local relations, moving along the $y$-axis, 
increases. Furthermore, the matrix identifies an area called ecological area. The clusters in this area are characterized by a medium-high strategic autonomy and a good networking capacity.

The last matrix evaluates the role of local system as enabler of long-term competitiveness. It is named the Local System Matrix. It evaluates the relevance of local system in term of economic coordination and sustainable development. The firms in a cluster compete globally through the mobilization of location-specific resource and the thickness of local system is seen as one of the most important variable to overcome the "globalisation trap" (Lagendijk, 2000). The matrix is defined by two dimensions:

- Sustainable Development: it is defined as average between strategic practices and business performances. In other word, the index evaluates the economic sustainability giving a score to the current firm's economic performances and to the strategic practice necessary to sustain it.

- Local System Efficiency: is defined as the weighted average between the perceived relevance of links with local context in comparison to the links with global system. The weighted average is based on four different type of connection (from the lowest to the highest): supplier market framework (4 items), low-service framework (3 items), high-service framework (3 items) and research and development framework (3 items).

Moving from left to right increases the local system efficiency and, thereby, the endogenous development processes. Therefore, the local context, moving along the $x-$ axis, changes from a status of institutional thinness to one of institutional thickness (Henry \& Pinch, 2001). Moving from bottom to up improves the sustainable development and, consequently, the level of strategic practices implemented and the level of business performances obtained. Therefore, moving along the $y$-axis changes the competitiveness foundations from a short-term view to a long-term.

\section{The empirical study on Emilia Romagna's cluster}

The empirical study concerns 7 clusters settled in Emilia Romagna. The main objective of this empirical study is twofold. The first is to test the suitability and appropriateness of the scoreboard. The second is to conduct a benchmarking study on the industrial clusters' competitiveness in the knowledge-based economy.

The empirical research was conducted as follow:

- Nine clusters in Emilia Romagna facing the challenges of global competition and knowledge economy were detected. These clusters are: automotive cluster in Bologna, biomedical cluster in Mirandola, ceramics Cluster in Sassuolo, footwear cluster in Fusignano and in San Mauro Pascoli, Packaging cluster in Val d'Enza, plastic cluster in Correggio and textile cluster in Carpi. We chose these clusters because they can be differentiated by sector and technology;

- Data were collected using a snowball methodology;

- A case history, based on the literature, for every cluster was written and a SWOT analysis, based on a interview with local policy makers and trade associations, was conducted;

- For every cluster the result of scoreboard were discussed in a focus group to verify the reliability of the model and the capability as policies' tool. The focus group participants are local policy maker, trade associations and some firms of the sample. 


\section{Research Sample}

When a study on a cluster is carried out it is difficult to choose the right subjects (respondents) to be interviewed because the structure of the network is not known in advance. Therefore, the snowball sampling method was chosen. According to Atkinson and Flint (2001) snowball sampling can be applied as a formal methodology for making inferences about hidden and/or hard-to-reach populations. Snowball method begins with a set of actors. The sample is created by asking every respondent to name one or more players who could contribute to the study. This process is based on the assumption that exist a link between the initial sample and the target population (Berg, 1988).

The initial set of actor was chosen selecting cluster's most relevant - in term of size, age and turnover - firms. The initial set was selected with the help of local policy maker and trade associations. Since we used snowball sampling method to asses the relationship and knowledge framework, we asked top ten supplier in terms of knowledge exchange and/or in term of relationship's quality. With this method it has been possible to interview both leader firms (typically in the initial set) and designers and subcontractor. The process stops when the sample realized cover all the filiére or the limits of time and resources were achieved.

The snowball method has problems of representativeness due to selection process of initial set (Atkinson \& Flint, 2001). The size of sample for every district should solve this type of problem. Furthermore it should be avoid to over-emphasize cohesiveness (Van Meter, 1990).

During the snowball process we contacted by telephone 700 enterprises and sent by e-mail the first part of questionnaire. A total of 497 questioners were returned $^{11}$. We start to collect data on nine cluster but in two of them (the shoe factory cluster of Fusignano and the automotive cluster of Bologna) the number of firms contacted was not large enough. Then 368 firms were interviewed for the second part of questionnaire.

The number of usable data for every cluster is shown in the Table 1.

Table 1 Size of realized sample and population for every cluster

\begin{tabular}{|l|r|r|r|r|}
\hline \multicolumn{1}{|c|}{ CLUSTER } & $\begin{array}{c}\text { SAMPLE } \\
(\mathrm{S})\end{array}$ & PERCENT & $\begin{array}{c}\text { POPULATION } 2003 \\
(\mathrm{P})^{*}\end{array}$ & $\frac{S}{P} \%$ \\
\hline BIOMEDICAL (MIRANDOLA) & 38 & $10,3 \%$ & 78 & $48,7 \%$ \\
\hline FOOTWEAR (S. MAURO PASCOLI) & 65 & $17,7 \%$ & 111 & $58,6 \%$ \\
\hline CERAMICS (SASSUOLO) & 54 & $14,7 \%$ & 1726 & $3,1 \%$ \\
\hline SHIPBUILDING (NORTH ADRIATIC) & 34 & $9,2 \%$ & 292 & $11,6 \%$ \\
\hline PACKAGING (VAL D'ENZA) & 69 & $18,8 \%$ & 458 & $15,1 \%$ \\
\hline PLASTIC (CORREGGIO) & 53 & $14,4 \%$ & 300 & $17,6 \%$ \\
\hline TEXTILE (CARPI) & 55 & $14,9 \%$ & 1483 & $3,7 \%$ \\
\hline TOTAL & 368 & $100,0 \%$ & $\#$ & $\#$ \\
\hline
\end{tabular}

Source: Istat, 2003

Broadly speaking, the seven cluster investigated were cluster of small and medium enterprise, settled in Emilia Romagna and operating in high-technology sector

${ }^{11}$ The response rate $(52,6 \%)$ is high because the enterprises were encouraged to completion by the possibility to participate at the Regional Excellence Award. 
(Biomedical cluster) or medium-technology sector (Ceramics, Packaging, Plastic and Shipbuilding cluster) or low-technology sector (Footwear and Textile Cluster) ${ }^{12}$.

Firms varied in size (Figure 1) and sales. Participant report a size ranging from 2 employees to 494 and turnover ranging from $€ 100$ thousand to $€ 98$ million annually. The firms were leaders $(89 ; 24,2 \%)$, designers $(101 ; 27,4 \%)$ and subcontractors (178; $48,4 \%)$. The Table 2 summarize the main characteristics of our final sample.

Although the sample is geographically constraint it is consistent to the objective of testing the scoreboard as a benchmarking tool. Indeed Emilia Romagna is considered one of the best Regional Innovation System (Braczyk, Cooke, \& Heidenreich, 1998) and it is located in the so called Third Italy (Bagnasco, 1977). Italian cluster are an industrial model based on a social network and on a mix of cooperation and competition (Pyke, Becattini \& Sengenberger, 1990). Now this industrial model is facing the challenges of globalization (Belussi \& Samarra, 2005) and the knowledge basedeconomy (Belussi \& Pilotti, 2002). Moreover the set of clusters used to test the framework are differentiated by technology level and industrial sector. Concluding, however the constraints, the sample is enough solid to test the scoreboard and to derive some interesting conclusions about the tool's capability.

${ }^{12}$ We consider the definition of OECD (1997) and Eurostat (2002) to define the level of technology. This classification is based on NACE Sector. 
Table 2 Main characteristics of final sample

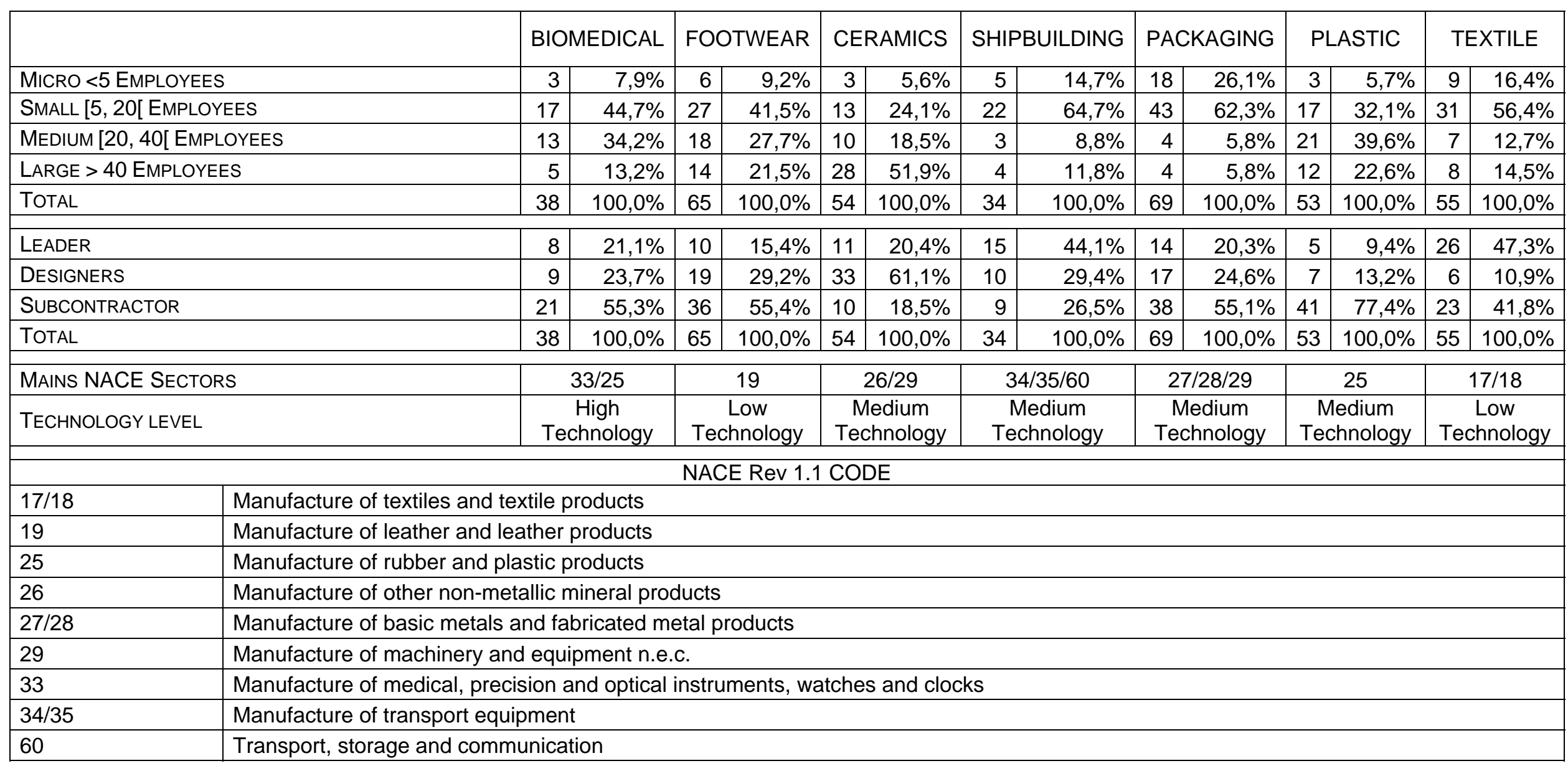




\section{Findings}

In this paragraph the scoreboard is presented and a benchmarking analysis is developed.

The Competitiveness Matrix (Figure 1) shows:

- The Ceramics Cluster, despite the level of technology and the global competition, has the best situation in term of network and in term of learning and knowledge;

- The others clusters, excluding the Plastic, are based on the same system of learning. However the Biomedical Cluster is placed in a worst position in term of quality of networking;

- The Plastic cluster is the worst on both dimensions.

The situation depicted by the first matrix it is interesting because Ceramics cluster and Plastic Cluster, though they have the same technology level and they are located in the same regional innovation system, have a completely different situation in term of competitiveness. The Packaging and the Shipbuilding clusters are also in a different situation. It confirms that the level of technology is not a good variable to define policies. Biomedical Cluster is in a unexpected position. This is a high-technology cluster; however the system of learning is not high. The second level of Scoreboard helps to understand the reasons of this position and to distinguish better the problems and the enablers of clusters' competitiveness.

Figure 1 The Competitiveness Matrix

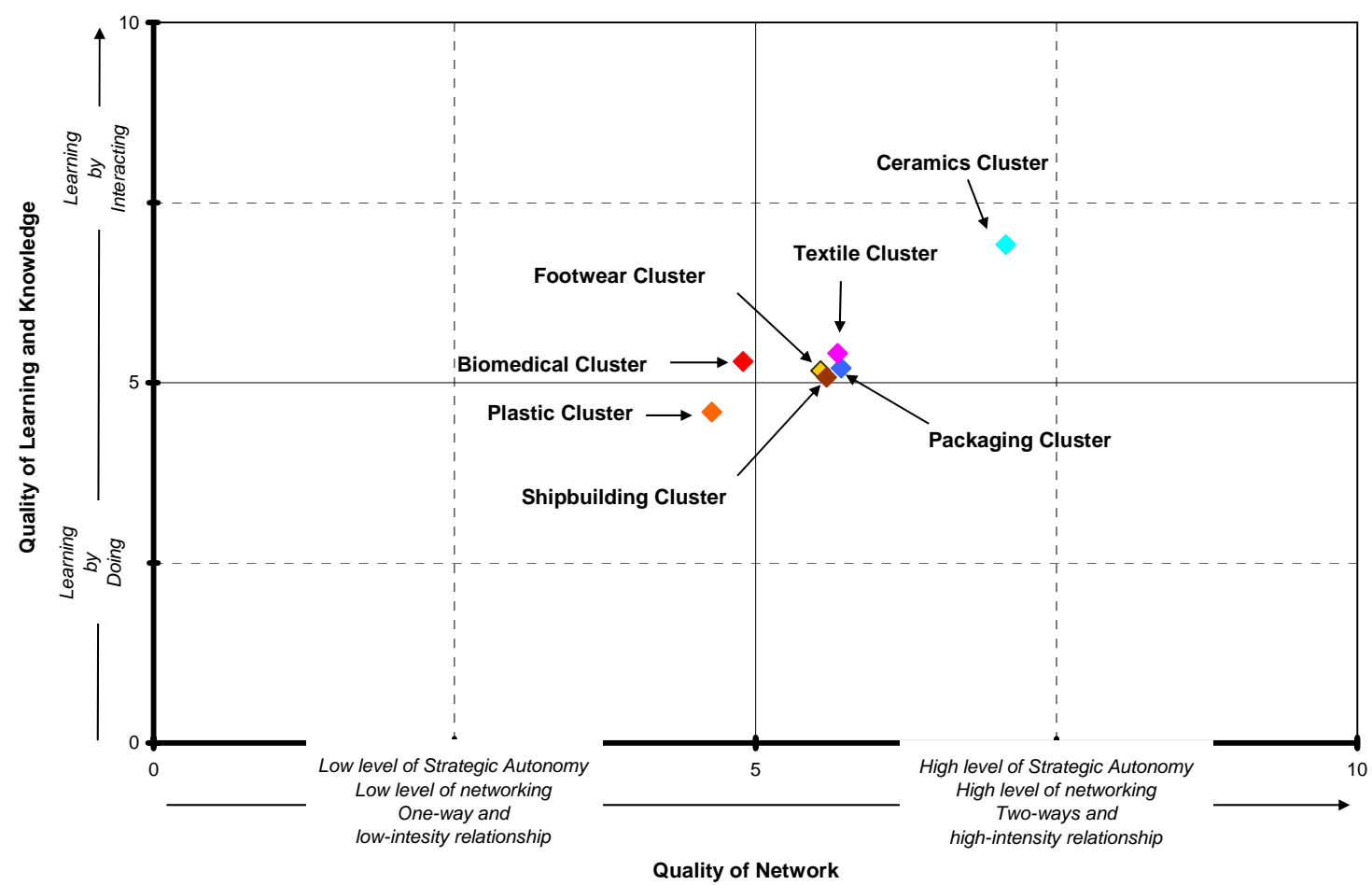

The Learning/Knowledge Matrix is useful to explore the differences between the cluster in terms of quality of learning and knowledge. The figure (Figure 2) shows that:

- The Ceramics Cluster is in the best position, the learning/knowledge framework of this cluster seems to be adapted to facing the challenges of the global economy; 
- The Biomedical and Shipbuilding cluster have a good system of learning; however, there is a lack in term of capabilities. These clusters point out a lack of an infrastructure capable of supporting collective learning and innovation. This situation is especially negative for Biomedical Cluster. In fact the Biomedical firms makes high-knowledge intensity activities but they do not activate a system of knowledge-networking;

- The Packaging, Textile and Footwear Cluster have a difficulty situation in terms of knowledge/learning and, particularly, in term of processes/activities internalized. It could be due to a high-dependence by external technologies or external market. For the Packaging cluster it could mean a specialization in outdated technologies. For the Textile and Footwear cluster the need to specialize on processes or activities with more value added. In both cases it requires to open up the networks to external actors and/or to mobilize the firm's complementary local asset (universities, trade unions, ...);

- The Plastic Cluster is in the worst position. It points out problems in the production and in the exchange of knowledge.

Figure 2 The Learning/Knowledge Matrix

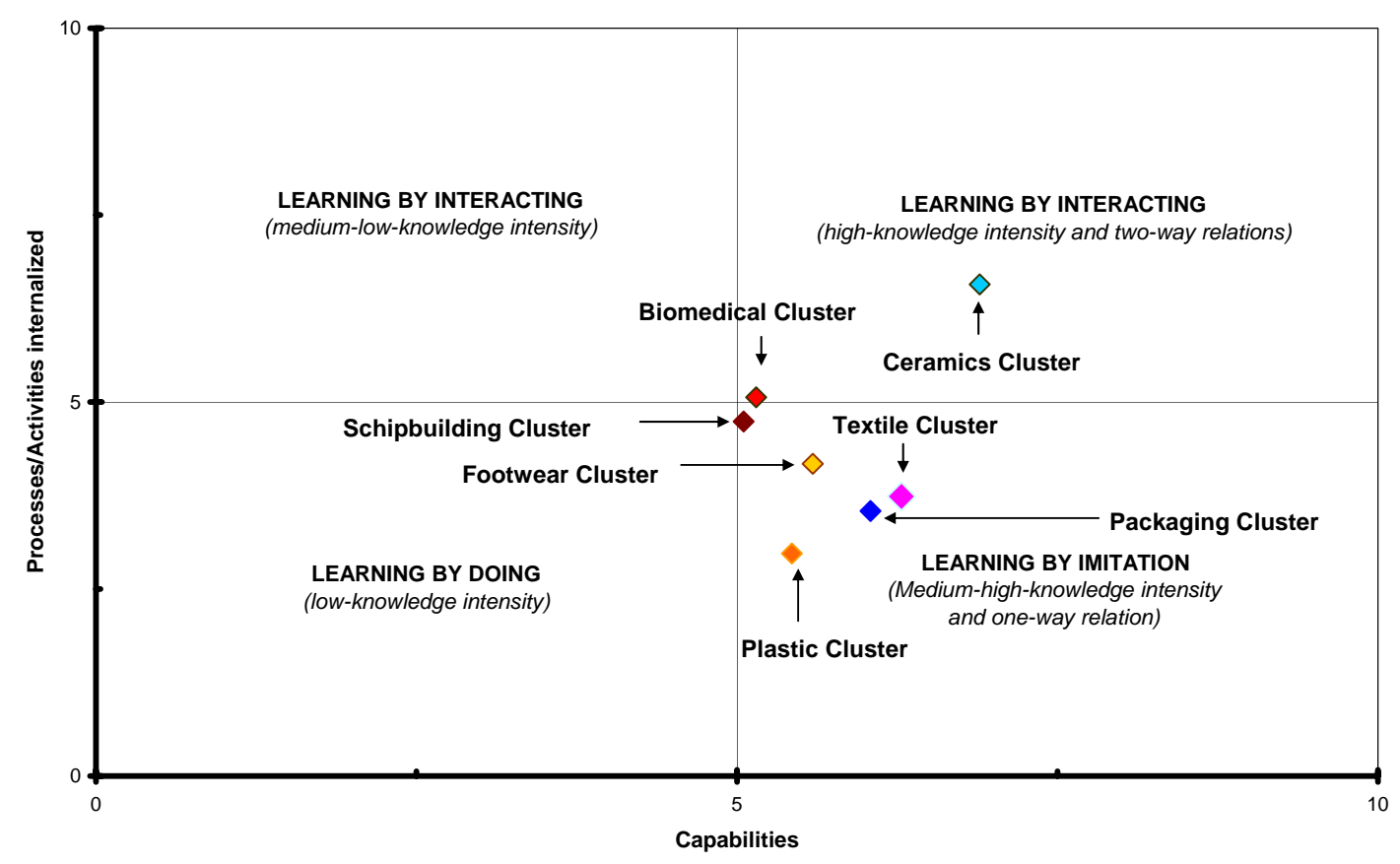

The Ecological Network Matrix enables us to explore the quality of network. The matrix (Figure 3) points out that:

- The Ceramics Cluster is characterized by a high strategic autonomy and a good networking of material and immaterial activities. It confirms the results of the previous matrixes;

- Packaging, Textile, Footwear and Shipbuilding Cluster have a balanced situation between the two dimensions. The first three need to mobilize the endogenous factors to improve the strategic autonomy in term of knowledge. The Shipbuilding cluster points out a lack of relational intensity probably due to the nature of the sector and the process of production. It reveals, another time, the need of an infrastructure capable of stimulate networking and learning;

- The Biomedical Cluster has a position that reveals two aspects. The first is the absence of a strong network. The second is the low level of strategic 
autonomy. The cluster's profile reveals a low use of knowledge. It could mean a lack of specialization on innovation activities, despite of the high-technology sector, in behalf of production activities. These are high-knowledge intensive; however without a renewing of these activities trough interaction the competitive framework of the cluster is not sustainable.

- According to previous matrixes, the worst position of Plastic Cluster. The firms in this cluster appear poor in term of strategic autonomy. The situation depicted until now shows the no-ecological competitive framework of this cluster. It competed using the endogenous resources without renewing it. Now it is not able to facing the global competitiveness.

Figure 3 The Ecological Network Matrix

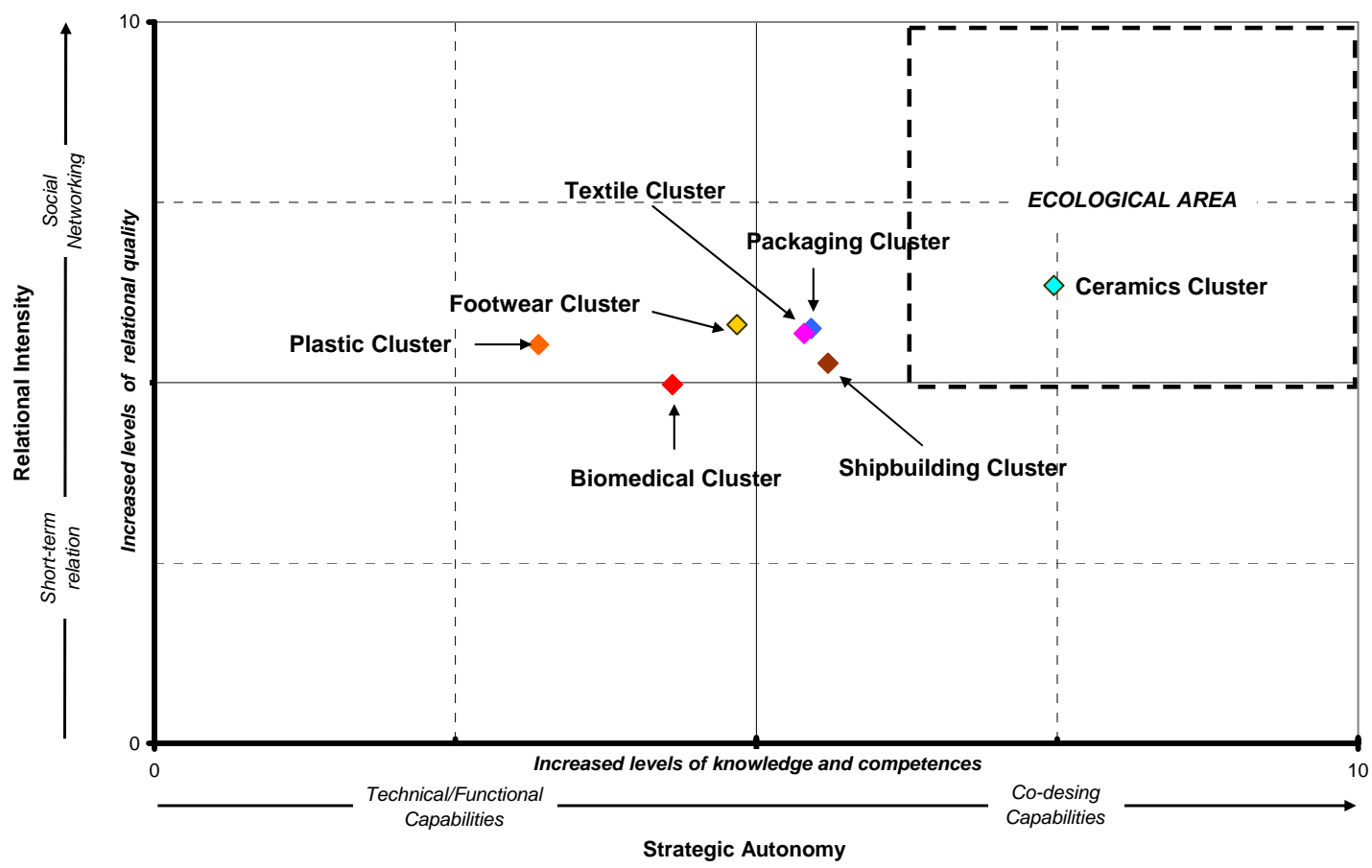

The Local System Matrix explores the role of local system as enabler of long-term competitiveness. The analysis of the matrix (Figure 4) points out three different situations:

- Plastic, Footwear, Packaging and Textile clusters do not find in the local context the right sustain to compete successful. This depiction points out a lack of relevant actors in the cluster. A good policies portfolio has to start from this type of problem. The most adequate approach may be link this cluster to external system to improve the general competitiveness foundations.

- Biomedical, Ceramics and Shipbuilding clusters have a good position in term of institutional thickness. Therefore to define the right policies portfolio it is enough to analyze the previous matrixes.

Figure 4 The Local System Matrix 


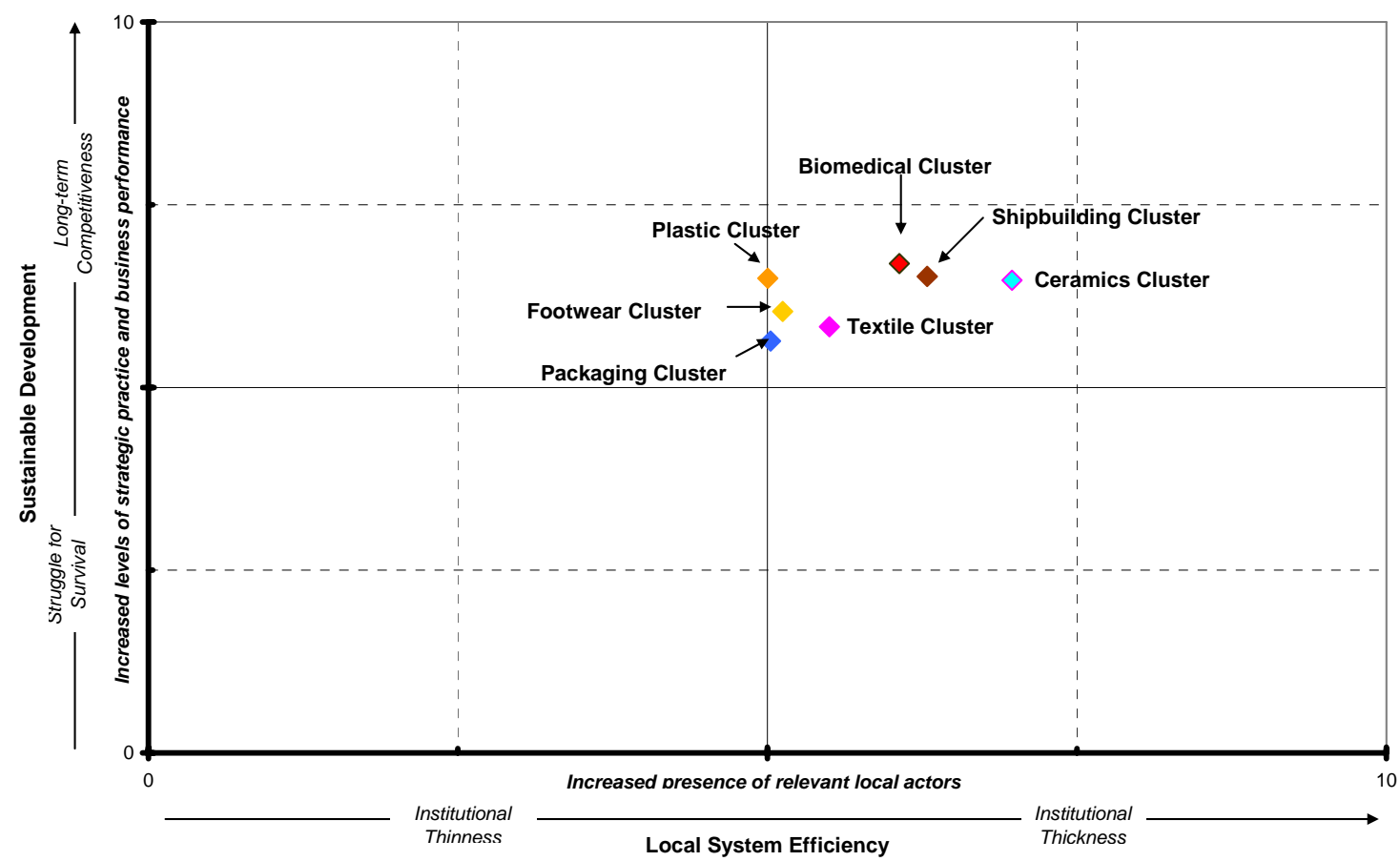

In conclusion, the application of the scoreboard to the seven clusters has permit to avoid policies misguiding due to false myths portfolio or success stories. In fact, the scoreboard assesses the presence of the most important requirements: the presence of a network, the system of learning and the presence of co-operation with local system. It permits to depict the follows situations:

- First of all an institutional thinness in Footwear, Plastic, Packaging and Textile cluster. Furthermore the matrixes points out that: in Packaging and Textile cluster is necessary to open up the networks to external actors and/or to mobilize the firm's complementary local asset (universities, trade unions, ...), in Footwear cluster is necessary to mobilize the endogenous factors to improve the strategic autonomy in term of knowledge, and finally in Plastic cluster is necessary to stimulate a renewing of endogenous resources;

- The need of an infrastructure capable of stimulate networking and learning and of supporting collective learning and innovation in Biomedical and Shipbuilding cluster;

- The good framework of Ceramics cluster. His competitive framework seems to be adapted to facing the challenges of the global economy. This cluster could represent a benchmark in term of policies and performances.

\section{Discussion and Conclusion}

\section{Summary}

In this paper we presented a possible scoreboard to measure clusters' competitiveness. The concept of competitiveness found its foundation in the strategic literature and mainly on Porter's work on firms' competitive advantage. It is Porter himself that, with his book on the competitive advantage of nations, exports the term competitiveness in regional economics. His major contribution is to highlight that firms' competitiveness does depend on the development of local resources. Even if an influential economist such as Krugman has strongly contrasted such a perspective because may lead to bad policy making, this concept has became, according to our perspective with reasons, to be part of our normal way of thinking. We argue, in fact, that the development of information, communication and transportation technologies, 
on the one hand, and the dematerialization of production on the other has intensified competition between regions on their capacity to attract, withhold and transform into local value the potential embedded in highly mobile and versatile global resources, such as knowledge and talent.

We look at cluster's competitiveness form a very specific perspective, which is the one ecology. The concept of ecology is not new to the strategic and managerial literature. It identifies a specific stream of studies aiming to prove the evolution is not the outcome of organizational change, but market selection. The basic idea is that successful firms accumulate an excess of organizational inertia that inhibits their internal capacity to change. Therefore, new technologies and organizational practices are brought to the market by new firms that outperform existing ones. We argue that the major limit of this perspective is that it does not take into account the network reactions and feedbacks that those changes enact. Taking this network into account enables to make creativity endogenous. It is not anymore sufficient to maximize 'firms' reproduction" in the hope that this will lead to a positive recombination of the original competitive factors, but is necessary to invest on the quality of environment (the cognitive infrastructure) to enhance firms' learning capacity.

Following Morin's contribution we derive a number of intrinsic characters that enhance ecologies' creativity. In other words, it enhance the capacity to sustain its development through the creation of new state of equilibrium. The most important are the capacity to integrate connectivity, resources diversity and openness, and strategic autonomy. The number of potential recombination, in fact, increases with the probability that diverse resources meet and generate and an innovative combination of resources. However, this is not enough. Resources' willingness and capacity (strategic autonomy) to explore their intrinsic combinatorial potential is determinant to make sure that creativity is maximized. Therefore, firms, in order to be strategically autonomous, are required to hold the necessary knowledge and competencies to explore its potential inside and outside the ecosystem.

\section{Limitations and future research}

Our work has also some limitations. The first is that our benchmarking tool does only focus on firms. However, the quality of the practices adopted by local institutions is also important. Furthermore, it is also important to know how these local institutions and associations have tried to support local firms, such as through founds devoted to stimulate innovation, internationalization and so forth. Therefore, future research will try to expand the current framework in order to include the assessment of the quality of the local institutional infrastructure. Furthermore, we are also planning to extend the use of these tools to the evaluation of other clusters in order to extend its validity to a crosscultural context.

\section{Contribution and implications}

In the last part of the paper we apply our scoreboard to a sample of seven clusters in Emilia Romagna. These clusters have been selected for their different degree of technological specialization (hi-tech versus low-tech). Our benchmarking analysis shows the existence of differential needs in each of the seven clusters. For instance, in the footwear, packaging, textile and plastic clusters the scoreboard signal the need to invest on improving the quality of the institutional context in order to enhance cooperation within clusters. In the shipbuilding and biomedical clusters the scoreboard highlights the need to enlarge the base of knowledge and competencies. We present our conclusions in local focus groups, where results have been found coherent with participants' knowledge and experience of their cluster. Therefore, our scoreboard proves itself useful to enhance local governance and strategic capacity. Furthermore, it may prove itself a useful framework to support policymakers in sharing their experiences across clusters. 


\section{References}

Arthur, W. B. (1996). Increasing Returns and the new world of business. Harvard Business Review, 74.4(Luglio/Agosto), 100-109.

Asheim, B. T., \& Isaksen, A. (2002). Regional Innovation Systems: The Integration of Local 'Sticky' and Global 'Ubiquitous' Knowledge. The Journal of Technology Transfer, 27(1), 77-86.

Bagnasco, A. (1977). Tre Italie: la problematica territoriale dello sviluppo italiano. Bologna: II Mulino.

Baum, J. A. C., Dobrev, S. D., \& van Witteloostuijn, A. (Eds.). (2006). Ecology and Strategy. Oxford: JAl/Elsevier.

Belussi, F. (2005). On the theory of spatial clustering: the emergence of various form of agglomeration. In F. Belussi \& A. Samarra (Eds.), Industrial District, Relocation, and the Governance of the Global Value Chain. Padova: Cleup.

Boschma, R. A. (2004). Competitiveness of Regions from an Evolutionary Perspective. Regional Studies, 38(9), 1001-1014.

Braczyk, H., Cooke, P., \& Heidenreich, M. (Eds.). (1998). Regional Innovation Systems. London: UCL Press.

Bruni, G. (2006). Reciprocità. Dinamiche di Cooperazione Economia e Società Civile. Milano: Mondadori.

Cooke, P., \& Huggins, R. (2002). High Technology Clustering in Cambridge. In A. Amin, S. Goglio \& F. Sforzi (Eds.), The Institutions of Local Development. London: IGU.

European Commision. (2002). European Innovation Scoreboard. Technical Paper, 2002(6).

Florida, R. (2002). The Rise of the Creative Class: And How It's Transforming Work, Leisure, Community and Everyday Life. New York: Basic Books.

Florida, R. (2005). The Flight of the Creative Class: The New Global Competition for Talent. New York: Harper.

Ganzaroli, A., \& Pilotti, L. (2006). L'Impresa dei Saperi tra Etica e Creatività verso Ecologie del Valore. In L. Pilotti \& A. Ganzaroli (Eds.), Rileggere il marketing Risorse informative e gestione della conoscenza. Padova: CEDAM.

Iansiti, M., \& Levien, R. (2004). Strategy as Ecology. Harvard Business Review, 82(3), 68-78.

Isaksen, A. (2001). Building Regional Innovation Systems: Is Endogenous Industrial Development Possible in the Global Economy? Canadian Journal of Regional Science, 24(1), 101-124.

Krugman, P. (1991). Increasing Returns and Economic Geography. Journal of Political Economic, 99(3), 483-499.

Krugman, P. (1994). Competitiveness: A Dangerous Obsession. Foreign Affairs, 73(2), 28-44.

Martin, R., \& Sunley, P. (2003). Deconstructing clusters: chaotic concept or policy panacea? Journal of Economic Geography, 1, 5-35.

Maskell, P. (2001). Towards a Knowledge-based Theory of the Geographical Cluster. Industrial and Corporate Change, 10(4), 921-943.

Morin, E. (1980). L'Ecologie Generalisee. Paris: Editinos du Seuil.

Newlands, D. (2003). Competition and Cooperation in Industrial Clusters: The Implications for Public Policy. European Planning Studies, 11(5), 521-532.

OECD. (1997). Revision of the high-technology sector and product classification. STI Working Paper, 1997(2).

Pilotti, L., \& Rinaldin, M. (2004). Culture and Arts as Knowledge resources towards sustainability for identity of nations. Finanza Marketing e Produzione, N. 1, Marzo.

Porter, M. (1990). The competitive advantage of nations. London: Macmillan.

Sen, A. (1995). Inequality Reexamined. New York: Oxford University Press. 